\title{
medicine
}

\section{Express delivery}

$\mathrm{T}$ he field of gene therapy has endured a roller-coaster ride of impending promise and grave setbacks. Despite the latter, experts remain optimistic that applied therapeutics for human disease are imminent. But to realize the full potential of gene therapy, there are some fundamental hurdles that must first be overcome.

In this month's issue, we present three articles that apply gene therapy in different models of disease and underscore the ongoing development of this field. First, Chao et al. modulate RNA processing in a mouse model of hemophilia $\mathrm{A}$. In this first in vivo application of spliceosome-mediated RNA trans-splicing, the researchers correct the mutant transcript and restore functional factor VIII expression. Lu et al. seek to restore muscle function in the $m d x$ mouse model of Duchenne muscular dystrophy, also using an RNA editing approach. In this case, an antisense oligoribonucleotide causes the mRNA splicing machinery to skip mutated exons in the dystrophin transcript. Haberman et al. use a more classical gene therapy approach in a rat model of epilepsy: an adeno-associated viral vector delivers a gene encoding the neuropeptide galanin, to control the excitatory-inhibitory balance in neurons. After a fibronectin secretory signal sequence was introduced into the galanin gene, the steady release of galanin from presynaptic vesicles attenuated seizures.

These articles illustrate the ever-expanding arsenal at researchers' disposal for the application of gene therapy. The new approaches stem from the exciting discoveries that continue to emerge from fundamental research on the molecular biology of RNA and DNA and the mechanisms that control gene expression. The expansion of the study of RNA interference, for instance, from the once seemingly esoteric phenomenon of plant viruses, exemplifies the dovetailing of disparate fields into therapeutic opportunities.

But the limiting factor that hampers the application of all these approaches is the pragmatic concern of gene delivery. The challenge of specifically and efficiently directing the therapeutic molecule to its intended site of action continues to impair effective translation from the laboratory to the clinic.

The critical importance of the vector system used in gene therapy is starkly illustrated by the recent tragedies in clinical trials. The death of Jesse Gelsinger in 1999 was directly attributable to the adenovirus vector used to deliver a functional ornithine transcarbamylase gene. Gelsinger suffered a massive inflammatory response to the adenovirus vector, resulting in his death. More recently, two children who had undergone gene therapy to correct the normally fatal X-linked severe combined immunodeficiency (XSCID) developed a leukemia-like disorder. The retroviral vector-used to introduce a functional, common cytokine receptor $\gamma$-chain gene into the patients' $T$ lymphocytes-had inserted into an oncogene, giving these cells a huge selective growth advantage and leading to the leukemia-like disease.

Before these terrible consequences, however, the XSCID trial had been considered a high point in gene therapy, bringing the field to a new and far more rigorous stage of classical clinical research. The patients had developed functional immune systems after reinfusion of engineered hematopoietic stem cells, and their disease had thus been 'cured'.

New modalities for safe and efficient gene restoration are now under active development, but chromosomal integration of the therapeutic effector molecules to provide persistent gene expression remains a key focus of these efforts. Viral vectors-in particular adeno-associated viruses and retroviruses-are still the most promising candidates for gene delivery. New serotypes of adenoassociated viruses with specific tropisms and increased efficiencies are under investigation, although improved gene-deleted or helper-dependent adenoviruses may also make a comeback. Nonviral vectors, as exemplified in the work of Lu et al., as well as viral-nonviral chimeric vectors are being developed, and transposons with site-specific integrases may refine chromosomal targeting. A wide variety of vectors that are inert and innocuous is needed; the ultimate choice will depend on the specific disease.

But vector-host interactions will continue to be an overriding concern. Complications due to immune responses to the viral capsid proteins and transgene products are the biggest obstacles, but other problems such as vector tropisms must also be addressed. Given that human outcomes rarely correlate with animal model data, this will require validation in human systems. Currently, these essential toxicology studies compete poorly for funding. The US National Institutes of Health and its counterparts elsewhere need to drive the research agenda, support innovative research into new vector systems and dedicate funds for the more mundane preclinical trials that are crucial to investigating vector potential in humans. Not until safe, efficient and specific delivery systems are in hand will gene therapy be able to realize all of its promise. 